NISTIR 6383

\title{
National Advanced Manufacturing Testbed
}

$A$ distributed testbed enabling collaborative research between scientists and engineers at remote locations.

\author{
David C. Stieren \\ Robert J. Densock \\ Mark E. Luce
}

\section{U.S. DEPARTMENT OF COMMERCE}

Technology Administration

National Institute of Standards and Technology

Manufacturing Engineering Laboratory

Office of Manufacturing Programs

Gaithersburg, MD 20899

August 1999

U.S. DEPARTMENT OF COMMERCE

William M. Daley, Secretary

TECHNOLOGY ADMINISTRATION

Cheryl L. Shavers, Under Secretary for Technology

NATIONAL INSTITUTE OF STANDARDS

AND TECHNOLOGY

Raymond G. Kammer, Director

$Q C$

100

.056

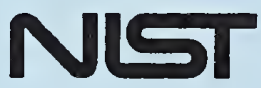

N0.6383

1999 



\title{
National Advanced Manufacturing Testbed
}

A distributed testbed enabling collaborative research between scientists and engineers at remote locations.

\author{
David C. Stieren \\ Robert J. Densock \\ Mark E. Luce
}

U.S. DEPARTMENT OF COMMERCE

Technology Administration

National Institute of Standards and Technology

Manufacturing Engineering Laboratory

Office of Manufacturing Programs

Gaithersburg, MD 20899

August 1999

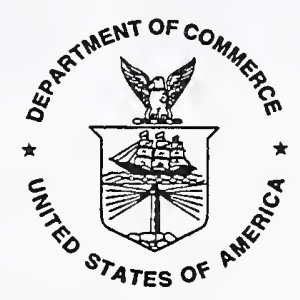

U.S. DEPARTMENT OF COMMERCE

William M. Daley, Secretary

TECHNOLOGY ADMINISTRATION

Cheryl L. Shavers, Under Secretary for Technology

NATIONAL INSTITUTE OF STANDARDS

AND TECHNOLOGY

Raymond G. Kammer, Director 



\section{Office of Manufacturing Programs}

The Office of Manufacturing Programs (OMP) manages cross-divisional and crosslaboratory, collaborative research and development programs for the Manufacturing Engineering Laboratory of the National Institute of Standards and Technology (NIST), including the Information-Based Manufacturing Program. The National Advanced Manufacturing Testbed (NAMT) is a distributed testbed managed by OMP that supports the activities of the Information-Based Manufacturing Program. The OMP plans and conducts facility upgrades, develops and coordinates technology demonstrations, and administers use of the resources and infrastructure supporting the NAMT. The OMP publishes formal progress reports for programs, conducts management reviews of project technical activities, plans and conducts technology demonstrations, and prepares program descriptive materials for public dissemination.

Staff points of contact within OMP for the NAMT are:

Mark Luce (Chief, OMP) mark.luce@ nist.gov

David Stieren (Program Manager) david.stieren@nist.gov

Robert Densock (Network Engineer) robert.densock@nist.gov

\section{Disclaimer}

No approval or endorsement of any commercial product by NIST is intended or implied. Certain commercial equipment, instruments, or materials are identified in this report in order to facilitate understanding. Such identification does not imply recommendation or endorsement by NIST, nor does it imply that the materials or equipment identified are necessarily the best available for the purpose.

This publication was prepared by United States Government employees as part of their official duties and is, therefore, a work of the U.S. Government and not subject to copyright. 



\section{Table of Contents}

Page

INTRODUCTION................................................... 1

Background................................................... 1

TESTBED OVERVIEW ................................................. 2

The NAMT Laboratory.......................................... 2

The NAMT Network Backbone.................................. 3

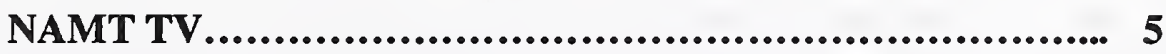

THE NAMT COLLABORATORY .................................... 7

Networking and Collaboratories................................. 9

NAMT ACCOMPLISHMENTS.......................................... 10

NAMT Design Characteristics...................................... 10

R\&D/Technical Capabilities Enabled............................ 13

Demonstrations Conducted....................................... 16

NAMT PLANS....................................................... 19

APPENDIX A: TESTBED COMPONENTS............................ 21

Computing Equipment in NAMT Laboratory.................. 21

Sample Software Applications..................................... 21

Networking Hard ware.............................................. 22

NAMT TV Carts................................................... 22

APPENDIX B: ORGANIZATIONS USING THE NAMT.............. 23

Representative Industry Organizations........................... 23

Representative Government Organizations........................ 23

Representative Academic Organizations............................ 24

REFERENCES.................................................. 25 



\section{INTRODUCTION}

The National Advanced Manufacturing Testbed (NAMT) is a distributed testbed enabling collaborative research between scientists and engineers at the National Institute of Standards and Technology (NIST), remote industry locations, and government and university laboratories. Since 1996 the NAMT has been the primary platform for enabling the operation of the Information-Based Manufacturing Programo of the NIST Manufacturing Engineering Laboratory (MEL). The NAMT supports the information technology (IT) needs of a collection of advanced research and development (R\&D) projects focusing on technology, measurement, and standards issues that are important to the industrial sectors served by NIST. Projects supported by the NAMT serve industries that are customers of the NIST MEL and the other NIST Measurements and Standards Laboratories, as well as the NIST Manufacturing Extension Partnership (www.mep.nist.gov) and Advanced Technology Program (www.atp.nist.gov).

The purpose of this report is to document the technological characteristics and functionality of the physical testbed components of the NAMT. The report documents the NAMT design rationale, including reasons why certain technologies and systems were selected to be part of the NAMT versus others.

\section{Background}

The NAMT was designed and developed to support the IT needs of R\&D projects being conducted at NIST and in partnership with organizations that are geographically located throughout the country and, in a few instances, the world. To best document how and why the NAMT has evolved into its present form, the two main areas with which the NAMT is concerned will be discussed: IT and R\&D.

IT within the context of the NAMT refers to those technologies that allow the electronic exchange of information. This definition of IT includes all the technologies and tools associated with networking systems, computing hardware, software systems, videoconferencing, faxes, telephones, pagers - basically, computing and electronic communications systems and technologies. The NAMT is a testbed where IT is primarily a support element that is applied, usually in a state-of-the-art, leading-edge manner, to the conduct of R\&D. During the course of applying IT to R\&D, developmental work that is specific to IT frequently occurs; however, the NAMT emphasis is mainly on the application of leading-edge IT to other domain-specific research areas.

NAMT-supported R\&D addresses a wide range of technology areas and scientific disciplines. Examples of these include:

- manufacturing technologies dealing with hardware and software issues relevant to products and processes

- electronic commerce issues 

- technologies for the automation of construction operations

- technologies associated with healthcare issues.

R\&D supported by the NAMT, regardless of the specific technical or industrial domain being addressed, is characterized by a focus on the development and application of technology, measurements, and standards. Additional information about the projects supported by the NAMT can be found in the NIST Internal Report, "Information-Based Manufacturing Program Summary and Accomplishments."(1)

\section{TESTBED OVERVIEW}

NAMT research partners, which are dispersed geographically about the country, conduct activities and share information between an array of organizations and physical facilities. To support the conduct of research, the NAMT supports information exchange between and among project partners through the application of advanced IT to projects. As such, the NAMT has been designed around an infrastructure for computing and communications whose foundation is a leading-edge, high-performance, network backbone connecting local project sites at high speeds and remote project sites via the Internet. The NAMT connects and supports state-of-the art computing systems that include high availability servers and high performance workstations, and projects exchange information over the NAMT infrastructure with collaborative, shared software applications that often have multimedia capabilities. The physical entities that constitute the NAMT infrastructure for computing and communications are described in the following sections.

\section{The NAMT Laboratory}

The NAMT Laboratory, located in the NIST Shops Building in Gaithersburg, Maryland, is logically at the center of the NAMT network backbone. Project laboratory sites at NIST and at partner locations can be accessed from the NAMT Lab in real-time for the exchange of voice, video, and data information. Access to the project laboratory sites means connectivity to the equipment, instrumentation, computing hardware and software, and scientists and engineers located in the labs. More information about this connectivity is provided in the section describing the NAMT network backbone.

Figure 1 is a diagram of the physical layout of the NAMT Lab. This is a working laboratory created to provide a state-of-the-art facility for computing and communications activities that are required to support the R\&D being conducted by projects. The NAMT Lab also frequently serves as a host facility for the conduct of technology demonstrations for projects.

The Lab contains an array of physical computing hardware systems connected to state-ofthe-art servers and consisting of several workstations, including Unix machines and highend desktop personal computers (PCs). Peripherals include both a color and black and 


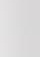


white printer, as well as a color plotter. The focal point of the laboratory is a highresolution, rear-projection system used for both research and demonstration purposes. This projection system is highlighted by a 90-inch screen mounted flush in the Lab's west wall. The projection system can show the display from any NAMT Lab computer, and the system can display high-resolution, live video images from the NIST laboratory sites of the projects supported by the NAMT, several NIST conference rooms, the NIST auditoriums, and several non-NIST partner sites. The live video images are transmitted over the NAMT network backbone as part of a system called, "NAMT TV," which is described in a later section of this document.

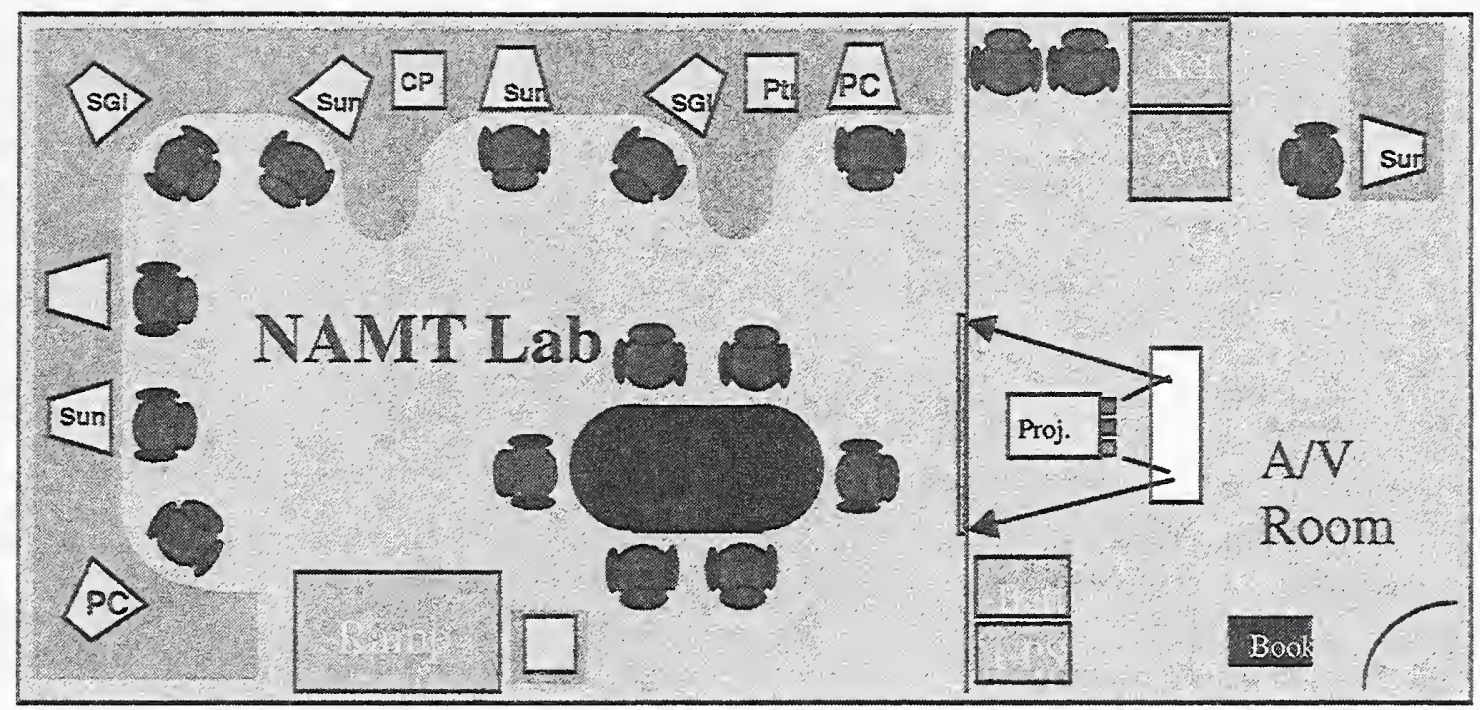

Figure 1. NAMT Lab

\section{The NAMT Network Backbone}

R\&D projects supported by the NAMT require high-performance network connectivity to perform many of the leading-edge activities that are part of their research applications. These network connectivity requirements exceed what can be achieved using the existing Internet. The NAMT network backbone allows, for example, live video images to be transmitted in full-motion, 30 frames per second capacity between laboratory sites that have connectivity. Video, audio, and data images can be communicated with negligible latency and with guaranteed quality of service. This basically means that if a communication session contains the transfer of information that requires some minimum amount of bandwidth, the NAMT network backbone technology is capable of providing that bandwidth without regard to any contention issues that are typical of multiple-user, broadcast environments with low bandwidth transmission limitations.

The network topology for the NAMT is depicted in Figure 2 on the next page. The figure shows the network connectivity for buildings and laboratories around the NIST campus 
in Gaithersburg, along with how this connectivity is extended to non-NIST locations in the wide area.

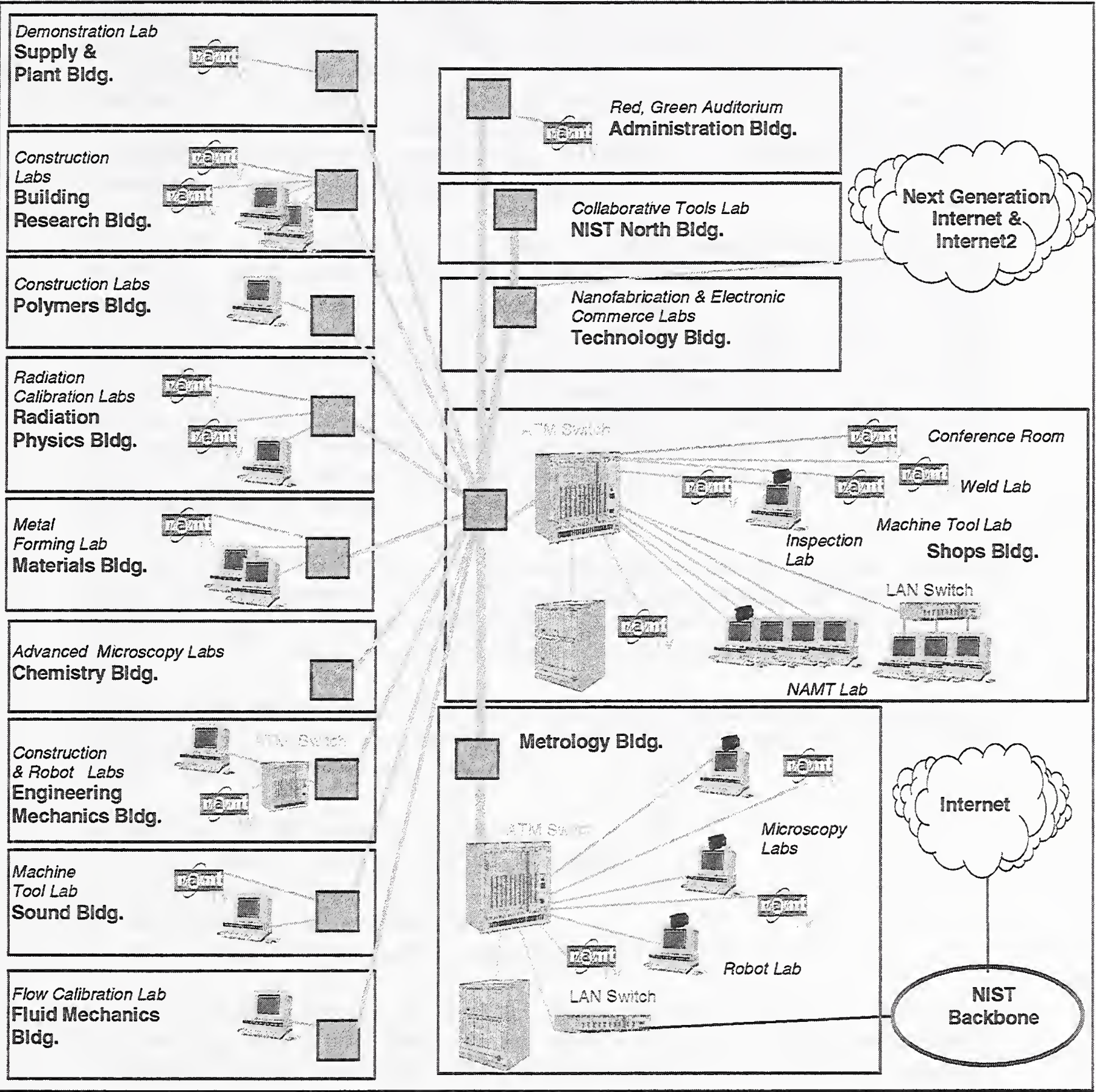

Figure 2. NAMT Network Topology 

Asynchronous transfer mode (ATM) is the primary networking technology used in the NAMT backbone, and ATM is the primary reason why the NAMT backbone operates with high performance. ATM is the first networking technology that was built from the ground up to handle the requirements of voice, video, and data over a single physical connection. Unlike other technologies, ATM creates dedicated, virtual circuits between pairs of communicating hosts. Virtual circuits are established with a guaranteed quality of service that allows the communicating hosts to exchange information without interruption from other parties. Constant-bit-rate applications like voice and video can be transmitted over an ATM network without breakups or jitter. When the pair of communicating hosts have completed their exchange, the ATM network releases the virtual circuit and the reserved network resources.

The NAMT ATM network was built using fiber optic cable as the physical medium. Each host connected to the ATM network has an OC-3c (Optical Carrier level 3) link that enables the host to achieve network transmission speeds of up to 155 Megabits per second (Mbps). The NAMT ATM network is used to connect high-performance workstations, enterprise servers, as well as special purpose audio/video encoders and decoders for real-time video conferencing between NAMT project sites. The NAMT network includes high-performance ATM connectivity to fourteen buildings and over 20 laboratory facilities, conference rooms, and auditoriums locally on the NIST campus. Over 100 non-NIST partner organizations connect to the NAMT with various levels of performance via the Internet.

The NIST NAMT network is part of the network backbone for the entire MEL, MELnet, which includes connectivity to over 400 workstations in labs and offices using category 5 cabling and switched Ethernet technologies. The MELnet provides connectivity to the desktops of MEL users with service bandwidths of $10 \mathrm{Mbps}$ to $155 \mathrm{Mbps}$.

The NAMT backbone serves the primary function of enabling partners to collaborate by accessing and leveraging resources in a high-performance manner. Remotely, NAMT partners can access the following resources:

- expertise of staff scientists and engineers

- highly capitalized or unique laboratory facilities

- computing and software systems

- testing and analysis equipment and data

- machine tools and manufacturing equipment and instrumentation

Several of these resources are depicted schematically in Figure 2 . The research capabilities associated with these resources, which are being both enabled and developed through the NAMT, are described in the NAMT Collaboratory section of this document.

\section{NAMT TV}

An innovative and practical means of access to and leverage of NAMT resources via the NAMT network backbone is through NAMT TV, which is seen in the NAMT network 

topology in Figure 2. NAMT TV is a portable, audio/visual (AV) system deployed using ATM networking that has been implemented as a means of minimizing and shielding the complexity of ATM technologies from the user. With NAMT TV, a series of mobile, self-contained AV carts are fielded at project laboratory sites around the NIST campus, and linked via ATM back to the NAMT Lab. This enables real-time, full-motion, twoway audio and video interactivity between project laboratory sites. NAMT TV systems are operated using simple, hand-held, remote controls that manipulate on-screen menu systems.

Each NAMT TV cart contains a video monitor and a series of microphones and cameras for sending and receiving audio and video signals to and from remotely located laboratory sites, including the NAMT Lab. These carts, which are hard-wired into the NAMT ATM network, send and receive signals using dedicated ATM encoders and decoders for signal compression and decompression, resulting in high-fidelity transmission and reception.

NAMT TV-enabled collaborative sessions between NAMT laboratory facilities send and receive full-motion video signals and high fidelity audio signals. NAMT TV sessions look and sound like two-way closed circuit television connections. The operational mechanics of a typical NAMT TV collaborative session are shown in Figure 3. NAMT capabilities have also been extended to several meeting rooms and auditorium facilities around the NIST campus as a means of conducting technical demonstrations for large and geographically distributed audiences.
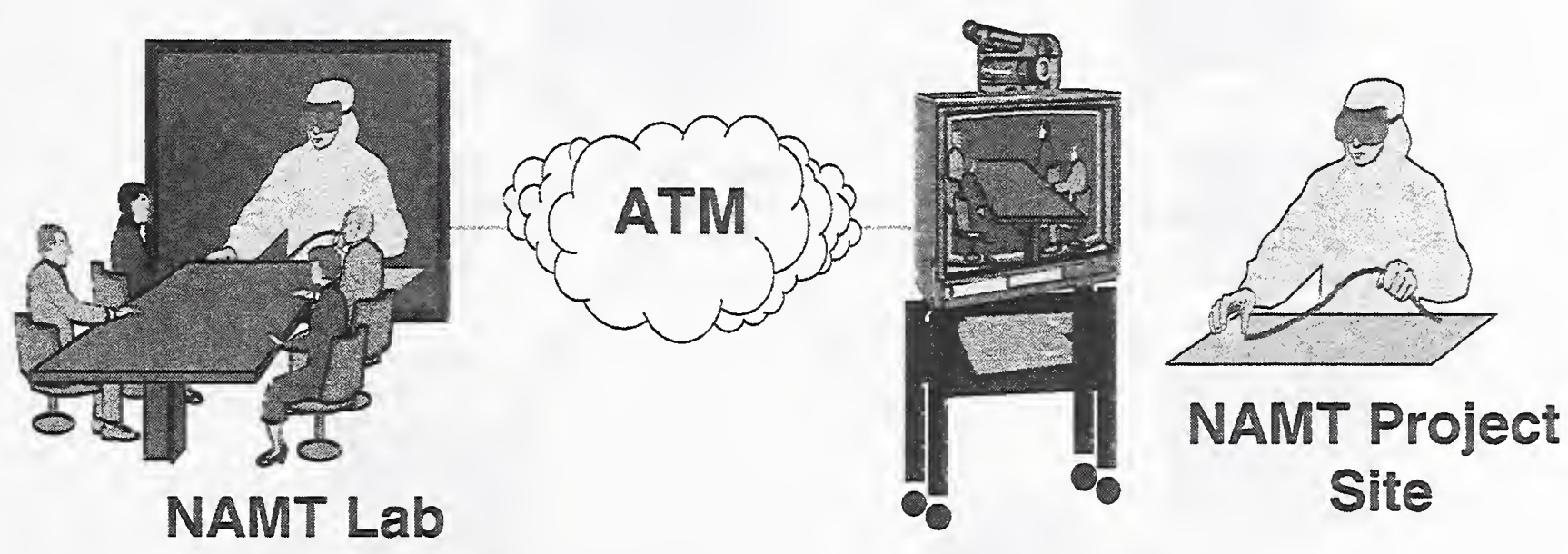

Figure 3. NAMT TV 

NAMT TV transmissions and collaborative sessions occur in the NAMT network backbone, where networking uses ATM technology end-to-end. This is true regardless of whether the transmission occurs in a local or wide area. The NAMT is supporting research efforts that are exploring the incorporation of NAMT TV signals into more widely accessible network environments that may not support ATM networking end-toend. Much of this R\&D is focused on developing a media gateway through which NAMT TV transmissions can be exchanged with typical Internet-enabled multimedia applications.

\section{THE NAMT COLLABORATORY}

The collaborative R\&D environment supported by the NAMT is similar in many ways to the concepts of a collaboratory, a term which is derived from a 1993 National Research Council (NRC) Report.2 A collaboratory can be considered as a "research center without walls." Within the NAMT collaboratory, various tools and systems that use computing and networking technology to aid manufacturing research are integrated together to provide an environment that allows scientists and engineers to make more efficient use of manufacturing resources, regardless of where they are located. The NAMT collaboratory structure is shown schematically in Figure 4.

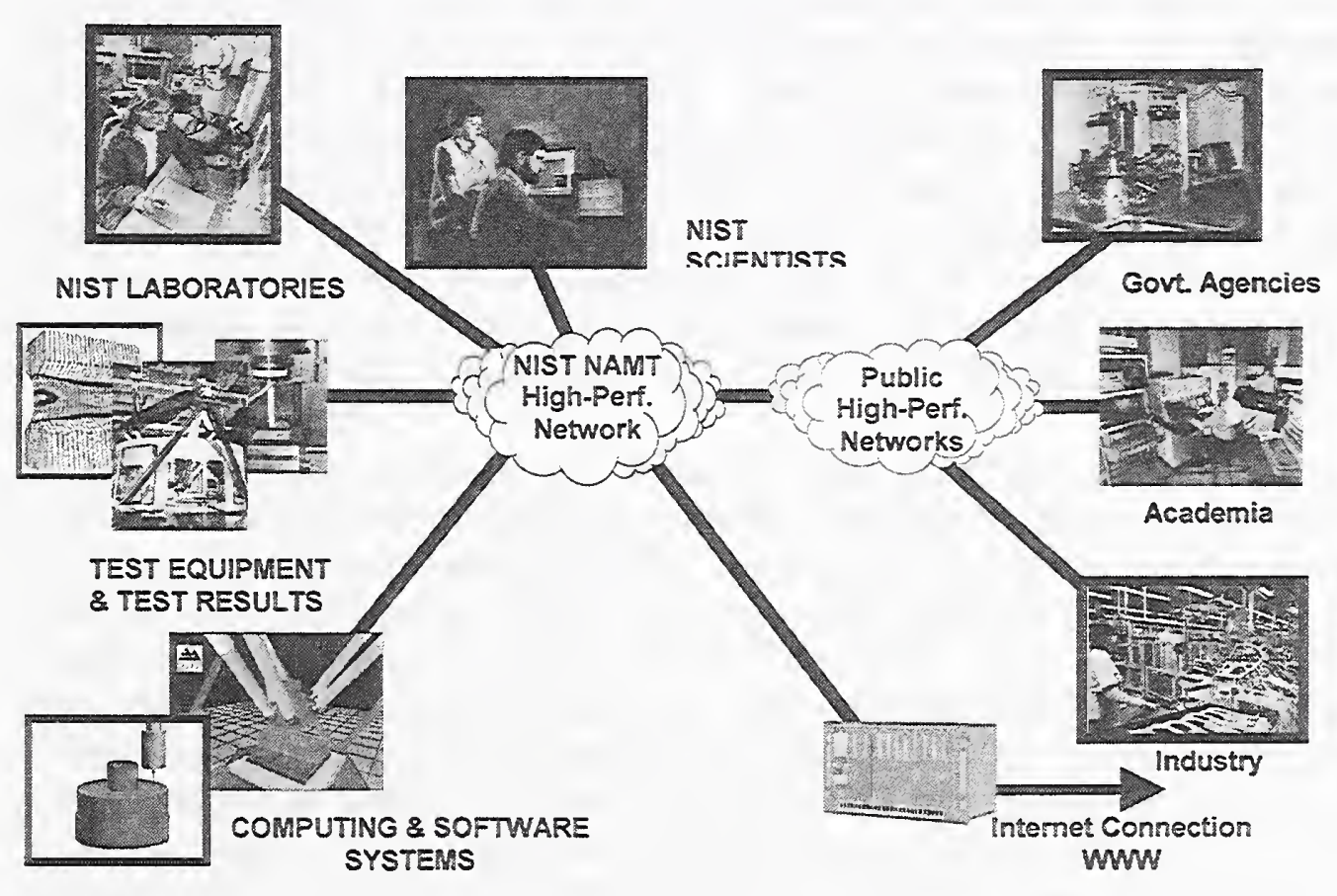

Figure 4. NAMT Collaboratory 

In the NAMT collaboratory, partners perform their research without regard to geographic location-interacting with colleagues, accessing equipment and instrumentation, sharing data and computational resources, and accessing information in digital libraries.

In the Information-Based Manufacturing Programo supported by the NAMT, advanced computing and communications capabilities are applied to manufacturing applications in order to link together facilities, equipment, staff and software, regardless of their physical location. The NAMT enables this to occur frequently and effectively through telepresence. NAMT TV, which was previously described, is a primary tool to enable telepresence.

Many definitions of the concept of telepresence have been developed over the past few years by people from a variety of business and technical backgrounds. One of the most concise definitions is contained in the 1993 NRC report on collaboratories:

“...telepresence...means that collaborators can work together from their own geographically distributed locations as effectively and efficiently as if they were physically co-located with one another."2

Telepresence can be important to manufacturing for several reasons, including the following:

- Telepresence provides the ability to leverage remotely located resources in a way that makes the remoteness of the resource location transparent to the organization(s) leveraging them. An example of this would be a situation where a company that manufactures equipment or instruments has a product in the field at a customer's location, which might be on the other side of the country. If the product were to fail and need repair, the manufacturer could access the product remotely in a matter of seconds through an on-line connection that would enable high-speed, secure transmission of voice, video, and data information. This could be done in such a way that diagnostics and trouble-shooting could be performed immediately, without lengthy travel delays.

- Telepresence also provides the ability to turn processes that involve multiple organizations and that are typically conducted sequentially into processes that can be conducted concurrently and efficiently. An example of this would be the design of a new product that involves teams of people from different facilities with different areas of expertise. Telepresence for concurrent design teams would allow multidisciplinary design teams to interact with one another from their home locations. These design teams could conduct all design activities on-line; instantaneously sharing expertise and information with one another from remote locations, providing team members access to information that might normally be "left back at the office" if the activities were to occur with everyone traveling to one location. This would also facilitate flexibility in scheduling collaborative activities, as travel schedules would not be an issue. 

Through telepresence, engineers and scientists have the opportunity to share information and data, to operate hardware and instrumentation, to diagnose problems, to develop programs, to conduct and analyze experiments, to develop designs - and do this jointly, from each participant's own geographic location.

The NRC telepresence definition implies many concepts regarding the achievement of telepresence. Among these are the following:

- real-time, two-way audio communications among collaborators

- real-time, two-way full motion video communications among collaborators

- real-time, two-way data communications among collaborators

The NAMT addresses the realization of telepresence through the network backbone previously described. As mentioned in this text, ATM provides network service at guaranteed high speeds with low latencies. Not all NAMT collaborators, however, have ATM connectivity, so the realization of telepresence for these applications is not comprehensive.

\section{Networking and Collaboratories}

Collaboratory operations and capabilities are closely dependent upon the level of sophistication and performance of the network connectivity that links together the collaborating facilities.

Connectivity to many remotely located NAMT partners now occurs through the Internet due to the relatively expensive costs associated with ATM connectivity and service. Much can be done with respect to the collaborative conduct of R\&D using today's Internet, but there are several limitations as well. Typically, Internet-enabled collaborations are limited by combinations of bandwidth, security, and quality of service. Large national efforts such as the President's Next Generation Internet (NGI) Initiative3 and other efforts supported by industry, government, and academia, including the Very High-Speed Backbone Network Service (vBNS)4 and Internet2 (I2)5 are in operation to advance today's Internet to a new generation of capabilities. Through these efforts, it is likely that the widespread use of high-speed, low-latency networks will very soon become reality in this country at affordable costs.

As of 1996 the Internet included more than 100,000 networks.4 These networks interconnect millions of computers and tens of millions of users throughout the world. The domestic portion of the Internet includes national backbone networks; regional and state networks; and networks at research, educational, government, and commercial institutions. Currently, research and educational institutions are usually connected to state or regional networks, which, in turn, are connected to either a regional network of broader extent or to a national backbone network. Backbone and other appropriate networks are interconnected at NAPs (Network Access Points), FIXes (Federal Internet eXchanges), the CIX (Commercial Internet eXchange), and various private exchange points. 

Information gathered in the development and operation of these efforts projects that the number of Internet users in the research and education community will continue to grow. It is also projected that these users will continue to require new levels of connectivity, performance, and services. New applications involving distributed, high-performance computing, remote visualization and imaging, and telecollaboration, together with growth in aggregate traffic, make the provision of an increasingly high-performance network infrastructure necessary.

With its array of advanced applications and many partners geographically distributed about the country, the information-based R\&D supported by the NAMT needs the availability of such a high performance network infrastructure. This will enable NAMT wide area network connectivity to operate at the performance levels required by the various research applications. As indicated in the NAMT network topology shown in Figure 2, the NAMT network backbone is capable of connecting to the emerging national high-performance networking systems being created, such as NGI and I2.

\section{NAMT ACCOMPLISHMENTS}

Technical activities associated with the NAMT began in January 1996 following an extensive planning and design period that covered several months and that involved expert input from a broad cross-section of manufacturing representatives from industry, government, and academia. These activities included the design and construction of the testbed and computing and communications infrastructure, as well as the R\&D activities of the projects the NAMT would support. Four advanced manufacturing R\&D projects began in 1996 that constituted the MEL Information-Based Manufacturing Program. (1) The IT requirements of these initial four projects, combined with input received in the planning process and estimates of future $R \& D$ needs, formulated the initial design of the NAMT.

The NAMT began with basically no usable network infrastructure in place in 1996. Since its inception, the NAMT has become a leading-edge testbed (as described in the Testbed Overview section of this report) that supports the IT needs of a collection of over a dozen advanced $R \& D$ projects.

\section{NAMT Design Characteristics}

To best detail the accomplishments of the NAMT, it is necessary to first describe how and why the NAMT was designed. It is important to reiterate here that the NAMT is the physical testbed and associated computing and communications infrastructure that supports R\&D within the Information-Based Manufacturing Program. (1)

IT by its nature changes and advances rapidly. The R\&D being conducted in the Information-Based Manufacturing Programo is both broad in scope and dynamic in 

nature. These facts drove the NAMT to be designed initially to embody attributes that would allow it to support the IT needs of R\&D projects and achieve the accomplishments described in this report. The attributes that were designed into the NAMT are summarized below:

- flexibility

- scalability

- accessibility

- utility

- reproducibility

\section{Flexibility}

Because it supports a diverse array of R\&D projects and technology requirements, the NAMT must be a flexible testbed with "plug-and-play" capabilities for technical applications. This means that hardware and software systems, projects, partners, computing and communications platforms, and sites can be added to and removed from the testbed without the need for customized interfaces. This also means that the R\&D conducted in the projects supported by the NAMT is amenable to change when dictated by industry needs. Additionally, new partners from industry, academia, and government can utilize the NAMT without major technical barriers preventing their entry.

This flexibility issue is addressed in the NAMT through the concerted attempt to avoid custom, point-to-point solutions for networking and computing. For example, although the NAMT uses ATM as its networking technology, it incorporates LAN emulation and Internet Protocols (IPs) as well. Flexibility is also addressed through the incorporation of hardware systems, software systems, and networking environments that have a wide range of complexity.

Specifically, the NAMT consists of the following components that contribute to its flexibility:

- computing hardware that ranges from PCs, to high-end Unix-based workstations and servers;

- software applications that range from simple Windows-based applications, to complex, numerically intensive graphics-based simulation and analysis programs; and

- a networking environment that ranges from Internet and Web-based capabilities, to high-performance [ATM] connectivity between various sites.

\section{Scalability}

To be scaleable, the NAMT must be able to change its size and scope as required by the projects it supports. Size here is measured in terms of organizational participation; number of projects; number of manufacturing equipment, devices, and instruments; number of computing platforms; number of communications devices; and number of 

software systems, tools, and applications. Scope is a function of project technical applications.

The IT systems employed in the NAMT-computing platforms, software applications, and network environments-have been implemented so that they are capable of accepting a diverse quantity of users and applications. In other words, the NAMT has been designed and implemented with capacity specifications that safely exceed project and application demands for all IT requirements. As one example of this, a NAMT TV session involving two-way interactivity and real-time, full-motion transfer of voice and video information between sites requires about $25 \mathrm{Mbps}$ of bandwidth. Such a session represents one of the most bandwidth-intensive communication connections currently occurring in the NAMT. The NAMT network backbone has capability to handle sessions requiring up to $155 \mathrm{Mbps}$ of bandwidth; thus, sufficient safety margin has been built in to ensure scalability.

\section{Accessibility}

Accessibility refers to the networking of heterogeneous computing and communications platforms, apparatuses, and technologies. This also refers to the sharing of software applications and data repositories by multiple, geographically-dispersed users, as well as remote access to manufacturing hardware. Perhaps most importantly, accessibility simply means the ability for users to operate the various systems that are networked together, regardless of the physical location of the users or the systems.

The NAMT addresses accessibility by providing access to systems based upon the least common denominator principal. This means that the infrastructure allows partners with little sophistication or few capabilities to be able to connect into the NAMT through simple, ubiquitous tools such as the Internet and Web browsers.

\section{Utility}

The NAMT employs high-performance ATM networking technology. ATM networking technology combines the best features of circuit-switched networks, like the telephone system, with the best features of packet-switched networks, like the Internet. ATM was built from the ground up to carry voice, video, and data traffic simultaneously at very high bandwidths over a single, physical network medium, providing capabilities that far exceed what can be achieved with the present day Internet.

The NAMT also uses standard Internet protocols to accommodate the needs of the majority of computing and network users today. This combination of high-performance networking with connectivity that adapts to the least common denominator and is based upon standard protocols provides an environment that proves useful to a very large crosssection of organizations, capabilities, and needs. 



\section{Reproducibility}

The mission intent of the NIST MEL calls for the measurements, standards, and infrastructure technologies developed in the R\&D supported by the NAMT to be capable of being applied (duplicated) in a widespread manner within the American industrial community. As such, to ensure the ability of NAMT results to be transitioned to industry, including spin off implementations of concepts, the NAMT was designed to be reproducible. A reproducible testbed is one that is based upon generic technologies that are standardized where appropriate. A reproducible testbed can be duplicated as necessary. Reproducibility implies the existence of public domain information that can be applied to any number of different specific uses. To this end, the MEL is striving to incorporate commercial technologies and recognized standards into the NAMT to the fullest extent.

\section{R\&D/Technical Capabilities Enabled}

The NAMT supports the IT needs of the Information-Based Manufacturing Programo R\&D projects. In fulfilling this IT support role, the NAMT has enabled or accelerated the development of new R\&D capabilities at NIST in conjunction this program.

Examples of a few of these technical capabilities and/or research areas are listed below, followed by a brief description of each. More information on these and the other NAMTsupported R\&D activities can be found in the NISTIR, "Information-Based Manufacturing Program Summary and Report."(1)

- Telemetrology for the on-line and collaborative conduct of NIST measurement and testing services

Several NIST measurement and testing services are being developed and conducted with the aid of telepresence where remotely-located organizations, including service customers, share resources in the conduct of measurements and tests. In some instance, this telepresence-aided resource sharing includes the teleoperation of remotely-located instrumentation and facilities by NIST scientists; and in other instances it means the on-line, often real-time, access to NIST facilities, instrumentation, or tests by NIST customers. The quality of remote access and level of capabilities achieved with respect to accessing remote resources - the extent to which telepresence is realized - is primarily a function of the networking capabilities available at the remotely-located, non-NIST organizations. The NAMT primarily provides the connectivity and develops and implements the associated capabilities that enable the telepresence for these efforts.

Telemetrology application areas include:

- micro- and nano-scale dimensional measurements, and physical and chemical analyses conducted by advanced microscopy instrumentation, including 

scanning tunneling microscopes, scanning electron microscopes, and atomic force microscopes

- measurement and testing of electrical standards and instrumentation, including digital multi-meters

- measurement of gas-flow properties and meters

- tensile testing for material analyses

- dosimetry measurement associated with radioactive sources and radiation processing

One example of how telepresence is being incorporated into NIST measurement services can be provided by a series of collaborative, digital multi-meter measurements that were performed recently. NIST frequently conducts international comparisons of its measurement capabilities in various areas with the national measurement institutes of other industrialized countries around the world. Recently in conjunction with the Organization of American States (OAS), NIST used NAMT telepresence capabilities recently to place on-line the calibration laboratories of eleven member nations of OAS. This facilitated the efficient, timely conduct of an international comparison for the calibration of digital multi-meter systems. The ability of the various countries to participate collaboratively in the measurement processes of each of the countries was successful in terms of reducing significantly the overall time required to conduct the multi-nation comparison. This also infused technology into those laboratories that needed it, facilitated training and education on world-class measurement procedures to the participating countries, and greatly facilitated information exchange and collaboration in an international environment.

- Shared, remotely-accessible, machine data repositories

NAMT-supported R\&D is developing model structures and repositories for housing performance information about various classes of manufacturing equipment, including machine tools and coordinate-based inspection systems. Information about these structures and repositories is exchanged electronically and can be accessed via the web. This R\&D is developing valuable tools to be used in the development of new product and process designs that accurately take into account actual manufacturing capabilities of producers and suppliers, offering the potential to reduce new product introduction times significantly. One NAMT industrial partner sees the availability of these tools as playing a significant role in helping them reduce by a factor of two the time it takes to go from major product design to completed production. The NAMT provides connectivity to the manufacturing facilities where the information for these models and repositories is collected. The NAMT also provides computing capabilities, facilities, and connectivity for developing the models and repositories. 

- Modeling and simulation tools for equipment and process characterization, including shared and web-accessible tools

NAMT-supported R\&D is developing methodologies for measuring, understanding, predicting, and enhancing the performance characteristics of various manufacturing equipment and processes. The NAMT supports this R\&D by providing much of the physical computing and networking to develop and share the computer-based models and simulation tools that assist this work. Several of the tools used in this research are accessible to collaborators via the web; others are accessible via highperformance network connectivity where available. A principle application area for this work is a new class of parallel-actuated machine tool known as an octahedral hexapod milling machine being studied at NIST and several interested manufacturing locations around the country. The capabilities developed here contribute to the development of a more in-depth understanding of process and equipment characteristics, as well as the ability to share this understanding across industries.

- Shared, web-accessible process monitoring and diagnostic tools

R\&D is underway to develop new capabilities for monitoring the status and quality of manufacturing operations in real-time from remote locations. This provides a means of bringing a particular operation to an expert, wherever that person may physically be, rather than transporting the expert to the operation. This concept is being developed to improve the means by which system and process diagnostics can be conducted for manufacturing operations. Ultimately, the aim is to reduce the time and cost it takes to conduct diagnostics and troubleshoot operations, and, in-turn, to reduce the time and cost associated with process development and equipment downtime. The NAMT is supporting the development of the tools necessary to achieve these capabilities, as well as providing the connectivity to the laboratories where the tools are being developed and the processes are being conducted. One example involves the remote process monitoring and diagnostics of robotic welding operations. Industries that conduct robotic welding frequently do so in a manner where the weld experts may be geographically separated from the systems doing the welding - either in a local sense within a factory location, or in a wide area sense, spanning multiple sites. The ability to bring the systems to the experts "instantaneously" over the web, regardless of the physical location of the expert or the system, allows experts to monitor operations and diagnose problems efficiently. This lends the potential to significantly reduce system and line downtime, as well as to reduce scrap and re-work due to incorrect welding parameters.

- Automated and remotely accessible construction and building operations

Capabilities are being developed for remotely monitoring, in real-time, the status of physical components that are found on construction sites. This R\&D is demonstrating the feasibility of automating and incorporating real-time metrology information into various construction operations. Information bases are being developed that model and track the dynamic status of construction components, from 

piles of dirt to backhoe or crane positions, often incorporating positioning data from global positioning systems. It is anticipated that the ability to model and track the components of job-sites comprehensively, combined with the ability to incorporate real-time metrology information into the automation of construction operations, will impact the time and cost of completion of construction jobs significantly. The NAMT is providing the networking capabilities for remote access between the various metrology, modeling, and positioning systems, as well as providing several computing facilities used in the research.

Additional construction research being supported by the NAMT is developing remotely-accessible, virtual testbeds for developing and testing control systems used in buildings. These testbeds provide neutral facilities for vendors and users of these systems to test the systems during their development, as well as prior to their fielded implementation.

- Distributed manufacturing supply chains conducting commerce electronically

NAMT-supported R\&D is demonstrating the feasibility of designing and manufacturing electronic components, including printed circuit and wiring assemblies, in supply chains that exchange information on-line. Design, manufacturing, and other commerce-related information is passed electronically among the various members of the supply chain, with each organization performing distinct design, manufacturing, and analysis tasks. The interactions are conducted via the Internet. The research is focusing on the development of the standards necessary to seamlessly pass the necessary technical and business information between supply chain organizations. This research and the capabilities being developed are considered to be representative of the future of how electronic commerce capabilities will affect manufacturing. Specifically, these capabilities are demonstrating how electronic commerce will enable enterprises to be integrated in both technical and business capabilities. The NAMT is providing the computing facilities and connectivity needed to demonstrate concept feasibility.

\section{Demonstrations Conducted}

The activities of the NAMT and the research it supports can be characterized as addressing leading edge technology issues and as being state-of-the-art within specific application domains. Many NAMT activities are oriented toward the demonstration of technical and concept feasibility, set within the context of the development of the appropriate measurements and standards to ensure widespread industrial implementation.

Due to the leading edge nature of its work and the orientation toward feasibility demonstration, much effort has been expended conducting technology demonstrations for audiences representing industry, government, and academic organizations. Since the commencement of technical activities in 1996, the NAMT has enabled the conduct of over 100 technical demonstrations. These demonstrations have involved extensive reliance upon the NAMT ATM network backbone, both locally and in the wide area, and 

the demonstrations have also involved capabilities that are enabled by commodity Internet networking.

Some of the more notable capability demonstrations that were conducted between 1996 and 1999, along with audiences who have received these demonstrations, are described here.

\section{Demonstrations Conducted at NIST}

- Technology/capability demonstrated: telemetrology for scanned probe microscopy

- demonstrated remote access to and control of NIST scanning tunneling microscopes for nano-scale metrology applications

- demonstration audiences included:

ㅁ The Honorable William Daley, U.S. Secretary of Commerce, and staff, 1997

- Deputy Secretary of Commerce Robert Mallet, 1998

․ Mary Good, Undersecretary of Commerce for Technology, 1997

a Cheryl Shavers, Undersecretary of Commerce for Technology (Mary Good's successor), 1999

a Donald Gips, Chief Domestic Policy Advisor to Vice President Gore, 1997

a 1997 National Manufacturing Technology Conference

a CAM-I Technical Advisory Board, 1998

a Staff members of the U.S. House of Representatives Science and Technology Committee, Technology Subcommittee, 1997

ㅁ The U.S. Senate Manufacturing Task Force, 1997

a National Science Foundation Engineering Directorate Program Managers, 1998

- Technology/capability demonstrated: collaborative R\&D for the characterization of new classes of manufacturing equipment

- Demonstrated remote access to and modeling and simulation tools for the NIST octahedral hexapod milling machine

- demonstration audiences included:

- The Honorable James Barcia and staff, U.S. House of Representatives, State of Michigan, 1998

a Donald Gips, Chief Domestic Policy Advisor to Vice President Gore, 1997

- 1997 National Manufacturing Technology Conference

CAM-I Technical Advisory Board, 1998

a Hexapod Users Group, 1997

- Staff members of the U.S. House of Representatives Science and Technology Committee, Technology Subcommittee, 1997

a The U.S. Senate Manufacturing Task Force, 1997

- National Science Foundation Engineering Directorate Program Managers, 1998 

- Technology/capability demonstrated: shared data repositories

- demonstrated capabilities to access and use data repositories and model structures for manufacturing equipment performance information

- demonstration audiences included:

a Donald Gips, Chief Domestic Policy Advisor to Vice President Gore, 1997

- 1997 National Manufacturing Technology Conference

a CAM-I Technical Advisory Board, 1998

- Staff members of the U.S. House of Representatives Science and Technology Committee, Technology Subcommittee, 1997

The U.S. Senate Manufacturing Task Force, 1997

- National Science Foundation Engineering Directorate Program Managers, 1998

- Technology/capability demonstrated: telemetrology for electrical standard calibration

- demonstrated the on-line collaborative calibration of digital multimeters in an international comparison setting

- demonstration audiences included:

- international representatives from 11 member countries of the Organization of American States involved in SIMNet, 1998

- Elliott Maxwell, Special Advisor to the U.S. Secretary of Commerce for the Digital Economy, 1999

- Technology/capability demonstrated: telemetrology for gas flow calibration - demonstrated teleoperation of gas flow meter calibration system at NIST

- 1998 international Tele-calibration of Flow Meters workshop hosted by NIST

- Technology/capability demonstrated: telemetrology for radiation applications

- demonstrated concepts for collaborative, automated dosimetry calibration services to be offered by NIST

- 1997 ionizing radiation measurements and standards workshop attended by international industrial and government organizations examining collaborative, automated dosimetry calibrations

\section{Demonstrations Conducted Off-Site from NIST}

- Technology/capability demonstrated: NAMT TV-enabled collaborative R\&D using area high-performance ATM connectivity between NIST laboratories in Gaithersburg, Maryland, and the remote demonstration locations

- demonstrated telepresence for and remote control of scanned probe microscopy

- demonstrated remote access to and simulation tools for the NIST octahedral hexapod milling machine

- demonstrated automated and remotely accessible construction operations

- demonstration events included: 

a Netamorphosis Next Generation Internet Initiative Technical Demonstration, Washington, D.C., a 1998 demonstration held for members of Congress, their staff, and members of the national media

- FOSE Federal Office Systems Expo, Washington, D.C., a 1997 information technology exposition for the federal government held at the Washington Convention Center

- Technology/capability demonstrated: Web-enabled collaborative R\&D using commodity Internet connectivity between NIST laboratories in Gaithersburg, Maryland, and the remote demonstration locations

- demonstrated shared, web-accessible process monitoring and diagnostic tools for remote, robotic arc-welding;

- demonstrated telemetrology capabilities for scanning electron microscopes

- demonstration events included:

- Federal Lab Technology Expo, Ford Motor Company Headquarters Technology Center, Dearborn, MI, a 1998 demonstration for over 1100 engineers and technicians from the Ford Motor Company and its suppliers

a Defense Manufacturing Conference, New Orleans, LA, a 1998 technical conference held at the New Orleans Marriott hotel highlighting Department of Defense manufacturing technologies, programs, and projects

- SC'98, Orlando, FL, a 1998 Supercomputing exposition held at the Orlando Convention Center

\section{NAMT PLANS}

The NAMT has successfully supported the IT needs of the R\&D projects in the NIST Information-Based Manufacturing Program since 1996, providing leading-edge IT capabilities to assist the conduct of the program's research. When the NAMT was created initially, it supported the needs of four projects in 1996, with that number growing in 1998 to its current portfolio of sixteen projects. The NAMT has been implemented in anticipation of and response to the needs of the R\&D it supports.

The future of the NAMT is basically a function of the nature and direction of future NIST R\&D, which is, in turn, a function of the needs of American industry. The NAMT will continue to support the IT needs of the sixteen projects it currently supports, likely for as long as these projects continue to operate as research efforts requiring support. Within the NIST MEL it is expected that the NAMT will play a more active role in supporting the IT needs of multiple strategic programs conducted by the Lab, in addition to the Information-Based Manufacturing Program.

Along with the support currently provided for these projects and described in this report, future NAMT support is likely to focus on: 

- the continued development and implementation of capabilities for wide area highperformance networking

- the infusion of high-fidelity, NAMT TV-like AV capabilities into IP-based communications

- close integration between the NAMT network backbone and the MELnet, providing leading-edge IT capabilities and support to the desktops of all MEL staff

All of the projects supported by the NAMT were planned as 3-5 year efforts, meaning that they are approaching the ends of their initial life cycles within the next few years. As such, the NAMT is destined for a transition in terms of the research it supports. The NIST MEL is presently in the process of planning the strategic R\&D programs it will be conducting in year 2000 and beyond - programs that very likely will rely upon the NAMT to provide IT support for their research needs. 



\section{APPENDIX A: TESTBED COMPONENTS}

The following summarizes the computing hardware and software and networking technologies that have been implemented in the NAMT, including NAMT TV systems. These lists are not necessarily comprehensive, but are provided with the intention of augmenting the information within the body of this report, indicating specific capabilities available in the Testbed.

\section{Computing Equipment in NAMT Laboratory}

- 2 Pentium II PC computers running Windows NT

- 2 SGI Indigo2 workstations with Max Impact graphics running IRIX 6.2 (one with ATM network interface)

- 1 Sun Ultra2 workstation with dual processors and Creator 3D graphics running Solaris 2.6

- 1 Sun Ultra1 workstation with Creator 3D graphics running Solaris 2.6 (with ATM network interface)

- 1 Sun SPARCstation 20 workstation running Solaris 2.6

- 1 Sun SPARCcenter 1000E with dual processors and 63GB of mirrored disk space

\section{Sample Software Applications}

- ProEngineer CAD system

- Deneb 3D simulation system

- Adams 3D simulation system

- Showme video conferencing system

- Netscape WWW browser

- Java development kit

- Centerline software development system

- Orbix CORBA communications system

- Matrix product data management system

- Object Store database management system

- CVW collaborative virtual workspace system

- Mathematica scientific computational system

- Steptools STEP development kit

- CADIQ CAD analysis system

- Teamwave collaborative software system

- Framemaker desktop publishing system

- Emacs text editor

- RealNetworks audio/video server and player 



\section{Networking Hardware}

- FORE Systems ASX-1000 10Gbps ATM backbone switch

- FORE Systems ES-3810 Workgroup Ethernet switch

- FORE Systems PowerHub 7000 Router

\section{NAMT TV Carts}

Each cart includes the following:

- Anthro cart with 19" rack section

- Sony 20" television

- Canon VC-C3 pan/tilt/zoom camera

- Shure audio mixer

- Lectrosonics wireless microphone (for scientist audio)

- Shure shotgun microphone (for scientific equipment audio)

- FORE Systems AVA-300 MJPEG over ATM encoder

- FORE Systems ATV-300 MJPEG over ATM decoder 



\section{APPENDIX B: $\quad$ ORGANIZATIONS USING THE NAMT}

The projects of the NAMT-supported NIST Information-Based Manufacturing Program are conducted in collaboration with organizations from U.S. industry, other government agencies, and universities. Collaboration on these projects assumes a variety of different forms, and several different vehicles are used to define organizational partnerships.

Project partners use the NAMT to leverage and access resources in the conduct of collaborative R\&D. How and the extent to which resources are leveraged and accessed, especially those that are physically located at NIST, are determined by several factors:

- the nature of the work being performed by a particular project,

- relevant technical issues, and

- relevant contractual stipulations that may be associated with a particular organization's partnership with a project.

The following organizations are partners in various NIST Information-Based Manufacturing Program projects. These organizations use the NAMT to access and leverage resources in various capacities. The lists below are not comprehensive, as only a half-dozen organizations representing industry, government, and academia are listed here. These lists are provided to indicate the types of organizations that use the NAMT as collaborators on NAMT-supported projects.

Additional information about partnerships associated with the NIST Information-Based Manufacturing Program can be found in the NISTIR, "Information-Based Manufacturing Program Summary and Report."(1)

\section{Representative Industry Organizations}

Caterpillar

Boeing

Honeywell / AMD

Raytheon

Ingersoll

United Technologies / Pratt \& Whitney

\section{Representative Government Organizations}

NASA Johnson Space Flight Center

Department of Energy Argonne National Laboratories

Department of Energy Sandia National Laboratories

Department of Energy Oak Ridge National Laboratories

Department of Energy Lawrence Livermore National Laboratories

Department of Defense Manufacturing Technology Program 

Representative Academic Organizations

University of Maryland

University of Florida

University of North Carolina-Charlotte

Georgia Polytechnic Institute

Carnegie Mellon University

Ohio State University 


$$
-
$$




\section{REFERENCES}

1. "Information-Based Manufacturing Program Summary and Accomplishments," Luce, M., and Stieren, D., NISTIR not yet published, U.S. Department of Commerce National Institute of Standards and Technology, Gaithersburg, Maryland, 1999.

2. $\quad$ National Collaboratories: Applying Information Technology for Scientific Research, Committee on a National Collaboratory, National Research Council Commission on Physical Sciences, Mathematics, and Applications, National Academy Press, Washington, D.C., 1993.

3. "Next Generation Internet Initiative" Presidential Advisory Committee on High Performance Computing and Communications, Information Technology, and the Next Generation Internet, National Coordination Office, http://www.ngi.gov

4. "Connections to the Internet," NSF Program Announcement 98-102, National Science Foundation Directorate for Computer Information Science and Engineering, http://www.nsf.gov/pubs/1998/nsf98102/nsf98102.htm

5. "Internet 2," University Corporation for Advanced Internet Development, http://www.internet2.edu 


